\title{
Effect of progesterone administration on the
} prognosis of patients with severe traumatic brain injury: a meta-analysis of randomized clinical trials

This article was published in the following Dove Medical Press journal:

Drug Design, Development and Therapy

\section{Zhi-Yong Pan \\ Yu-Hang Zhao \\ Wen-Hong Huang \\ Zhi-Ze Xiao \\ Zhi-Qiang Li}

Department of Neurosurgery,

Zhongnan Hospital of Wuhan

University, Wuhan, Hubei, PR China

Correspondence: Zhi-Qiang Li

Department of Neurosurgery, Zhongnan Hospital of Wuhan University, Donghu

Road 169, Wuhan 43007I, PR China

Tel +8627 678I 3005

Fax +86 27 678I 2892

Email lizhiqiang@whu.edu.cn
Purpose: The aim of this study was to assess the neuroprotective effect of progesterone administration on severe traumatic brain injury (TBI) for different follow-up periods and administration route by completing a meta-analysis of randomized clinical trials (RCTs).

Methods: A systematic literature search of PubMed, Embase, and Cochrane databases and the Web of Science (from establishment of each to September 1, 2018) was performed to identify original RCTs that evaluated the associations between progesterone treatment and the prognosis of patients with severe TBI.

Results: Eight RCTs enrolling 2,251 patients with severe TBI were included. Within 3 months post-injury, patients with progesterone administration had a lower mortality (risk ratio $[\mathrm{RR}]=0.59 ; 95 \% \mathrm{CI}[0.42-0.81], P=0.001)$ and better neurologic outcomes $(\mathrm{RR}=1.51$; $95 \%$ CI [1.12-2.02], $P=0.007)$ than those who received placebo. However, these differences did not persist at 6 months post-injury for mortality $(\mathrm{RR}=0.96 ; 95 \% \mathrm{CI}[0.65-1.41], P=0.83)$ or neurologic outcomes $(\mathrm{RR}=1.09 ; 95 \% \mathrm{CI}[0.93-1.27], P=0.31)$. The analysis stratified by administration route showed that beneficial effects were only observed in patients who received progesterone intramuscularly ( $\mathrm{RR}=1.61,95 \% \mathrm{CI}[1.19-2.18], P=0.002)$; no benefit was observed with intravenous administration ( $\mathrm{RR}=0.99,95 \% \mathrm{CI}[0.91-1.07], P=0.75)$.

Conclusion: Progesterone administration improved the clinical outcomes of severe TBI patients within 3 months but may not have significant long-term benefits 6 months post-injury.

Keywords: progesterone, severe traumatic brain injury, neuroprotection

\section{Introduction}

Traumatic brain injury (TBI) is an alteration in brain function resulting from an external mechanical force, such as rapid acceleration or deceleration, blast waves, crush, object impact, or penetration. ${ }^{1}$ TBI survivors may suffer temporary or permanent cognitive, physical, and psychosocial impairments, which impose significant emotional and economic burdens. ${ }^{2}$ TBI is therefore considered a serious worldwide public health problem.

Many experimental studies in the last few decades have greatly improved our understanding of the neuronal dysfunction mechanisms after TBI. There has also been considerable progress in new clinical therapies for TBI. Based on the gender difference in response to brain injury, some have proposed that female sex hormones are neuroprotective. One group reported that female TBI patients have better outcomes in work capacity and suggested that this may be due to progesterone. ${ }^{3}$ This hormone is produced not only by the ovaries and placenta but also by glial and neuronal cells of the central nervous system (CNS). ${ }^{4,5}$ Progesterone has neuroactive and neurosteroidal action in the CNS, and its neuroprotective effects have been shown in a variety of

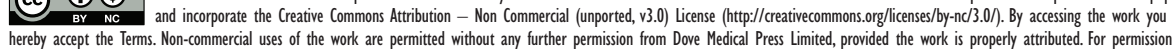
for commercial use of this work, please see paragraphs 4.2 and 5 of our Terms (https://www.dovepress.com/terms.php).
s. 
preclinical and clinical studies. Progesterone decreases cerebral edema, ${ }^{6,7}$ restores blood-brain barrier integrity, ${ }^{8,9}$ reduces the inflammatory response, ${ }^{10}$ and prevents cellular necrosis and apoptosis. ${ }^{11,12}$ In a systematic review of controlled animal studies, progesterone administration before or after acute cerebral injury had a dose-dependent effect on reducing lesion volume following TBI. ${ }^{13}$ Several randomized clinical trials (RCTs) have evaluated the efficacy of progesterone in treating TBI. A randomized, double-blind, placebo-controlled trial including 100 adult patients demonstrated a lower 30-day mortality rate in the intravenous progesterone group and good outcomes for patients with moderate injury. ${ }^{14} \mathrm{~A}$ similar conclusion was reached by another intervention review of RCTs. ${ }^{15}$ However, the results of two randomized, double-blind, multicenter Phase III trials published in 2014 evoked controversy regarding the efficacy and safety of progesterone. ${ }^{16,17}$ Those trials found no significant difference between placebo- and progesterone-treated groups in regard to functional outcome and mortality rate at 6 months post-TBI.

Patient outcome in a clinical trial may be affected by confounding factors. For example, the follow-up periods and drug delivery routes varied in these RCTs. The end point of follow-up was defined as 6 months in some clinical trials, compared to 30 days or 3 months in others. Progesterone was administered by intravenous infusion, intramuscular injection, or gavage. Whether these factors explain the discrepant clinical benefits of progesterone is unclear. Therefore, we conducted this meta-analysis of RCTs to assess the neuroprotective effect of progesterone in patients with severe TBI. This is the first investigation of the impact of follow-up period and administration route on the efficacy of progesterone for severe TBI.

\section{Methods}

\section{Search strategy}

A literature search was performed by two independent investigators to identify RCTs from PubMed, Embase, Cochrane Library, and the Web of Science (publications from each database up to September 2018 were considered). We also checked the bibliographies and references through a manual search of citations from all relevant publications to identify other potentially eligible studies. The search strategy employed the following keywords: progesterone or estrogen and traumatic brain injury or cerebral injury or brain injury.

\section{Inclusion and exclusion criteria}

Studies meeting the following criteria were included: 1) study design: published RCTs; 2) participants: patients $>15$ years old clinically diagnosed with acute severe TBI and a Glasgow Coma Scale (GCS) score $\leq 8 ; 3$ ) intervention: progesterone vs no progesterone or placebo administered in any dose, by any route, for any duration of time initiated within 24 hours of TBI; and 4) primary outcomes: mortality and neurologic outcomes. Neurologic outcomes were evaluated according to the Glasgow Outcome Scale (GOS). GOS scores were dichotomized into favorable outcomes (moderate disability and good recovery: GOS 4 and 5) and unfavorable outcomes (death, vegetative state, and severe disability: GOS 1-3). Studies were excluded for the following reasons: 1) studies enrolling pediatric patients, 2) studies only containing abstracts without full text, 3) studies lacking adequate original data, or 4) patients with GCS score $>8$.

\section{Quality assessment}

Two investigators independently assessed the eligibility and quality of the included studies and resolved any disagreement by discussion. The quality of each RCT was evaluated by assessing the risk of bias caused by random sequence generation, allocation concealment, blinding of participants and personnel, blinding of outcome assessment, incomplete outcome data, and selective reporting. The risk of bias for each domain was assessed as "low risk," "unclear risk," or "high risk."

\section{Data extraction}

Two investigators independently extracted the following data using standard data tables: first author, publication year, study design, participant demographics, diagnostic criteria of severe TBI, progesterone administration (drug, dose, and duration), and outcome variables. We contacted authors through e-mail to obtain key study details when needed. Any disagreements were resolved by discussion.

\section{Quantitative data synthesis}

Mortality and neurologic outcomes were chosen as the primary end points. Secondary outcomes were intracranial pressure, GCS score, and adverse effects. These outcomes were analyzed on an intention-to-treat basis. We pooled study results of the common risk ratio (RR) and risk differences with a 95\% CI using a Mantel-Haenszel model. ${ }^{18}$ Heterogeneity among studies was evaluated by Cochrane $Q$ tests and $I^{2}$ statistics. If significant heterogeneity was shown ( $P>0.1$ or $I^{2} \leq 25 \%$ ), a fixed-effects model was selected; otherwise, a random-effects model was used. Based on the Cochrane recommendation, heterogeneity was classified as low $(\leq 25 \%)$, moderate $(25 \%-50 \%)$, or high $(>50 \%)$. We calculated the presence of publication bias using funnel plots. 
Statistical analysis was performed using the Review Manager 5.1 software (Cochrane Collaboration, London, UK).

\section{Results}

\section{Characteristics of the included studies}

Initially, 528 articles were screened, and eight articles including 2,251 patients were eventually identified. ${ }^{14,16,17,19-23}$ The flow chart of literature selection is shown in Figure 1. The characteristics of the eight trials included in this analysis are presented in Table 1 .

\section{Risk of bias in the included studies}

The risk of bias in the included studies is shown in Figure 2. Three $e^{14,16,17}$ provided the information about all the domains of bias. One study ${ }^{22}$ provided all details except reporting bias. Three single-blind studies ${ }^{20,21,23}$ did not provide information regarding performance bias. One study ${ }^{19}$ did not report the details of allocation concealment, blinding method, or selective reporting.

\section{Association between progesterone treatment and mortality rate}

Four studies ${ }^{14,19-21}$ reported mortality within 3 months postinjury, three ${ }^{16,17,23}$ reported the mortality at 6 months post-injury, and one ${ }^{22}$ reported the mortality at both time points (Table 1 ).

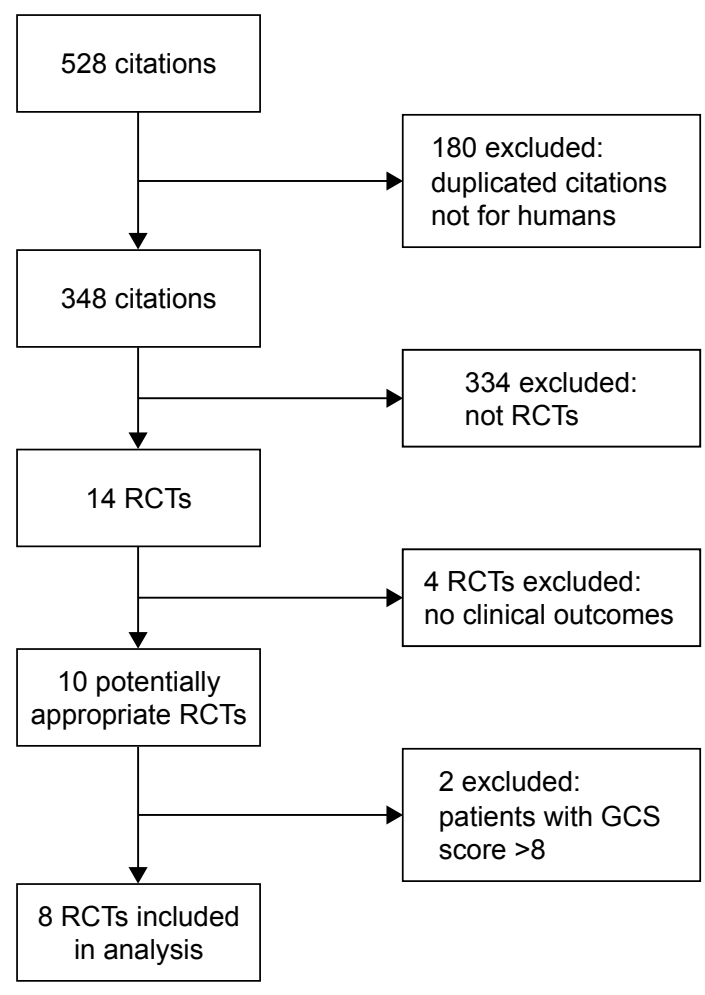

Figure I Flow diagram of study selection.

Abbreviations: GCS, Glasgow Coma Scale; RCT, randomized clinical trial.
We then investigated whether progesterone treatment had similar effects on mortality at different follow-up periods. There was no substantial evidence of heterogeneity among studies concerning mortality rate within 3 months $(P=0.68$, $I^{2}=0 \%$, Figure $3 \mathrm{~A}$ ). The mortality rate within 3 months in progesterone-treated patients was significantly lower than that in patients given placebo $(\mathrm{RR}=0.59 ; 95 \% \mathrm{CI}[0.42-0.81]$, $P=0.001)$. Pooled analysis showed moderate heterogeneity concerning mortality at 6 months post-injury $(P=0.10$, $\left.I^{2}=52 \%\right)$. No significant difference in mortality was observed between the progesterone and placebo groups at 6 months post-injury ( $\mathrm{RR}=0.96 ; 95 \% \mathrm{CI}[0.65-1.41], P=0.83$, Figure 3B).

\section{Association between progesterone treatment and neurologic outcomes}

Neurologic outcome was assessed by GOS score. Of the included studies, the one by Xiao et al was excluded because only mean GOS scores were reported in the study, and the data were insufficient for analysis. We did not observe substantial evidence of heterogeneity among the trials concerning GOS score within 3 months post-injury $(P=0.58$, $I^{2}=0 \%$ ). Patients treated with progesterone had better neurologic outcomes within 3 months post-injury than those given placebo $(\mathrm{RR}=1.51,95 \% \mathrm{CI}[1.12-2.02], P=0.007$, Figure 4A). High heterogeneity was observed among trials concerning GOS scores at 6 months post-injury $(P=0.06$, $I^{2}=60 \%$ ), and similar neurologic outcomes were observed for both the progesterone and placebo groups $(\mathrm{RR}=1.09$, $95 \%$ CI [0.93-1.27], Figure 4B).

\section{Effect of drug administration route on clinical outcomes}

Progesterone was administered intravenously in three studies, ${ }^{14,16,17}$ intramuscularly in four, ${ }^{19,20,22,23}$ and medroxyprogesterone tablets were taken by gavage in one study ${ }^{21} \mathrm{We}$ further evaluated whether route of delivery affected clinical outcomes in severe TBI patients. Our analysis showed that the mortality rate of patients with intramuscular administration was significantly lower compared to placebo $(\mathrm{RR}=0.61$, 95\% CI [0.41-0.92], $P=0.02$, Figure 5A), but this difference with the placebo group was not observed for patients given intravenous progesterone ( $\mathrm{RR}=0.96,95 \% \mathrm{CI}$ [0.63-1.46], $P=0.86$, Figure 5A). Similar results were observed for neurologic outcomes. The beneficial effect of progesterone on neurologic outcomes was only observed in patients treated with intramuscular progesterone $(\mathrm{RR}=1.61,95 \% \mathrm{CI}$ [1.19-2.18], $P=0.002$, Figure 5B), but not in patients given progesterone intravenously $(\mathrm{RR}=0.99,95 \% \mathrm{CI}$ [0.91-1.07], $P=0.75$, Figure 5B). 


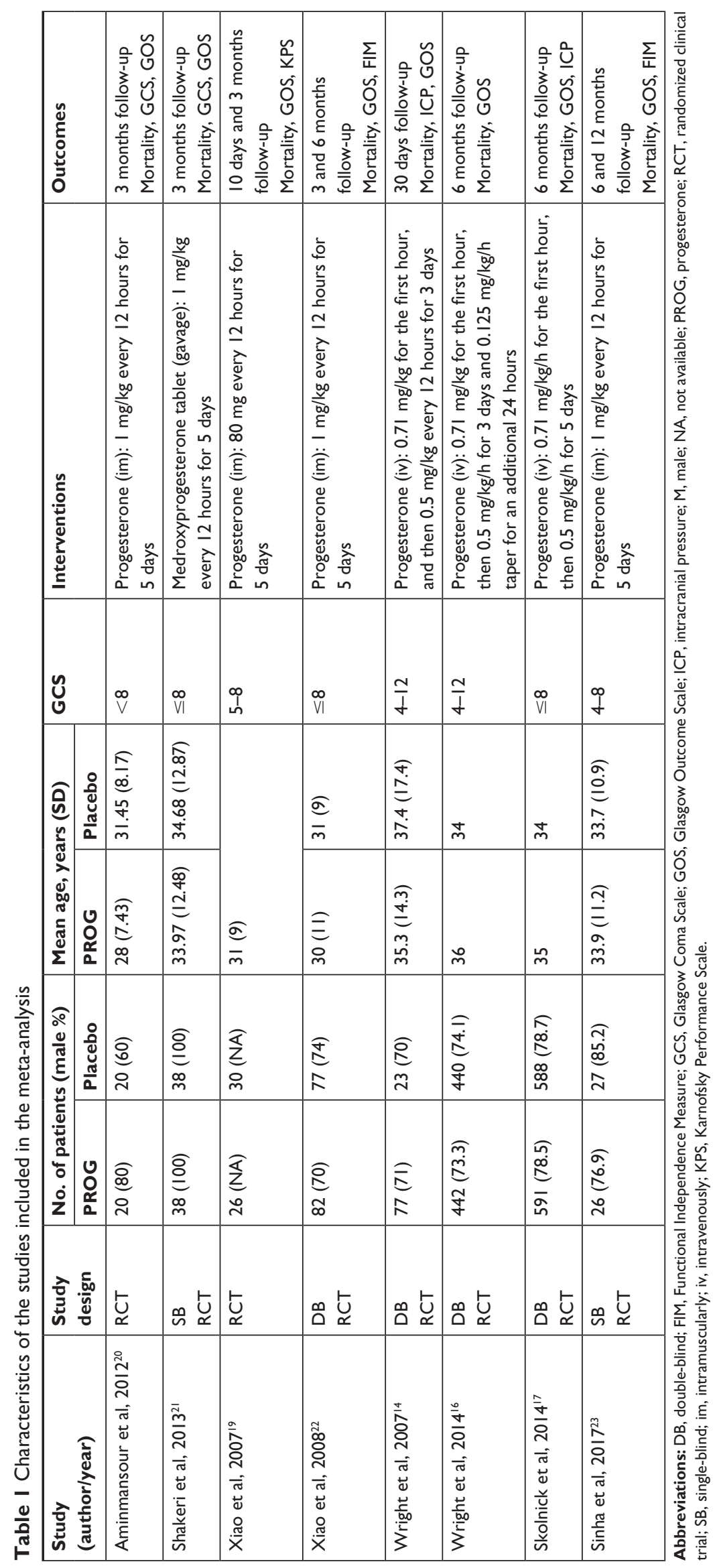




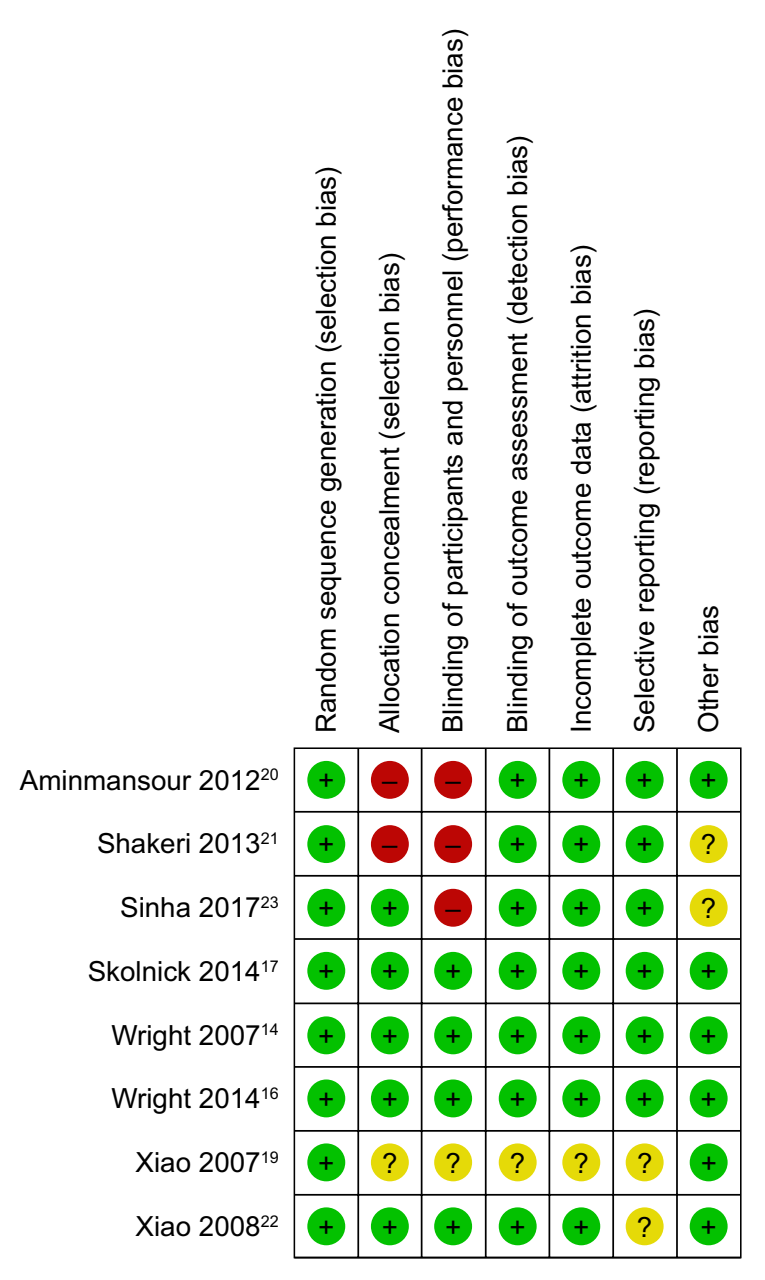

Figure 2 Risk of bias in each included study.

\section{Sensitivity analysis}

Sensitivity analysis was completed by the sequential elimination of each study. A significant difference was observed for neurologic outcomes within 3 months post-injury. When we excluded Xiao et al's study, which had a heavy weight of $52.7 \%$, the overall effect was reversed ( $\mathrm{RR}=1.48 ; 95 \%$ CI [0.96-2.29]), and the results were no longer significant $(P=0.08)$. This indicates that the result for neurologic outcomes within 3 months post-injury was not very robust.

\section{Discussion}

This meta-analysis aimed to evaluate the efficacy of progesterone in reducing the mortality rate and promoting neurological rehabilitation after severe TBI. The results indicate that progesterone could improve both primary outcomes in the short term (within 3 months after injury) but not in the long term (6 months post-injury).

Many studies have shown that progesterone exerts neuroprotective properties in animal models and clinical trials of TBI, with no serious adverse effects related to treatment. ${ }^{12}$
In TBI animal models, progesterone's neuroprotective properties are closely associated with a reduction of secondary injuries after TBI. Progesterone decreases cerebral edema and prevents secondary neuronal degeneration, thus ameliorating behavioral impairments caused by TBI. ${ }^{24}$ Two independent Phase II clinical trials reported beneficial effects of progesterone on mortality and GOS score in TBI patients. ${ }^{14,22}$ However, two randomized, double-blind, multicenter Phase III RCTs concluded that there was no significant difference between placebo- and progesterone-treated groups in regard to mortality rate and functional outcome at 6 months post-TBI. ${ }^{16,17}$ We noticed that these two studies evaluated clinical outcomes at different time points. Shortterm mortality and neurological outcomes are predominantly associated with the severity of primary and secondary brain damage, while the long-term status of patients with TBI may also be affected by factors such as complex complications, subsequent rehabilitation, comorbid conditions, and varied therapies. A 2016 meta-analysis showed that progesterone did not decrease mortality or improve neurological outcome in severe TBI patients. ${ }^{25}$ Seven RCTs ${ }^{14,16,17,19-22}$ included in our study were also assessed in that meta-analysis. The follow-up period in these trials ranged from 30 days to 6 months, but there was no analysis stratified by follow-up period, so timedependent effects of progesterone were not considered. We evaluated the efficacy of progesterone in severe TBI patients at short-term (within 3 months post-injury) and long-term (at 6 months post-injury) end points. Interestingly, progesterone administration was associated with a lower mortality rate and higher GOS score within 3 months post-injury, but no significant benefit was observed at 6 months. However, the long-term effects of progesterone should be interpreted cautiously in light of many confounding factors. Mortality and GOS score are commonly used to evaluate the clinical outcome of severe TBI patients. However, recovery from severe TBI is a slow, gradual process. Mortality and GOS score are not sufficiently sensitive to quantitatively measure functional deficits and gradual recovery over time. ${ }^{26} \mathrm{In}$ animal models, progesterone effects were evaluated by TBI-related pathological changes, such as brain edema, cell apoptosis, excitotoxicity, and inflammatory response in the brain. These are difficult to measure in clinical trials. When recovery is only modest, outcome improvement is not sensitively reflected by mortality or GOS. In addition, the end points selected for evaluation may also affect clinical trial results. ${ }^{26}$ It is unknown as to how long the injured brain takes to heal or if assessments at 3 or 6 months post-injury are sufficient to judge its recovery. Future studies should include longer 
A

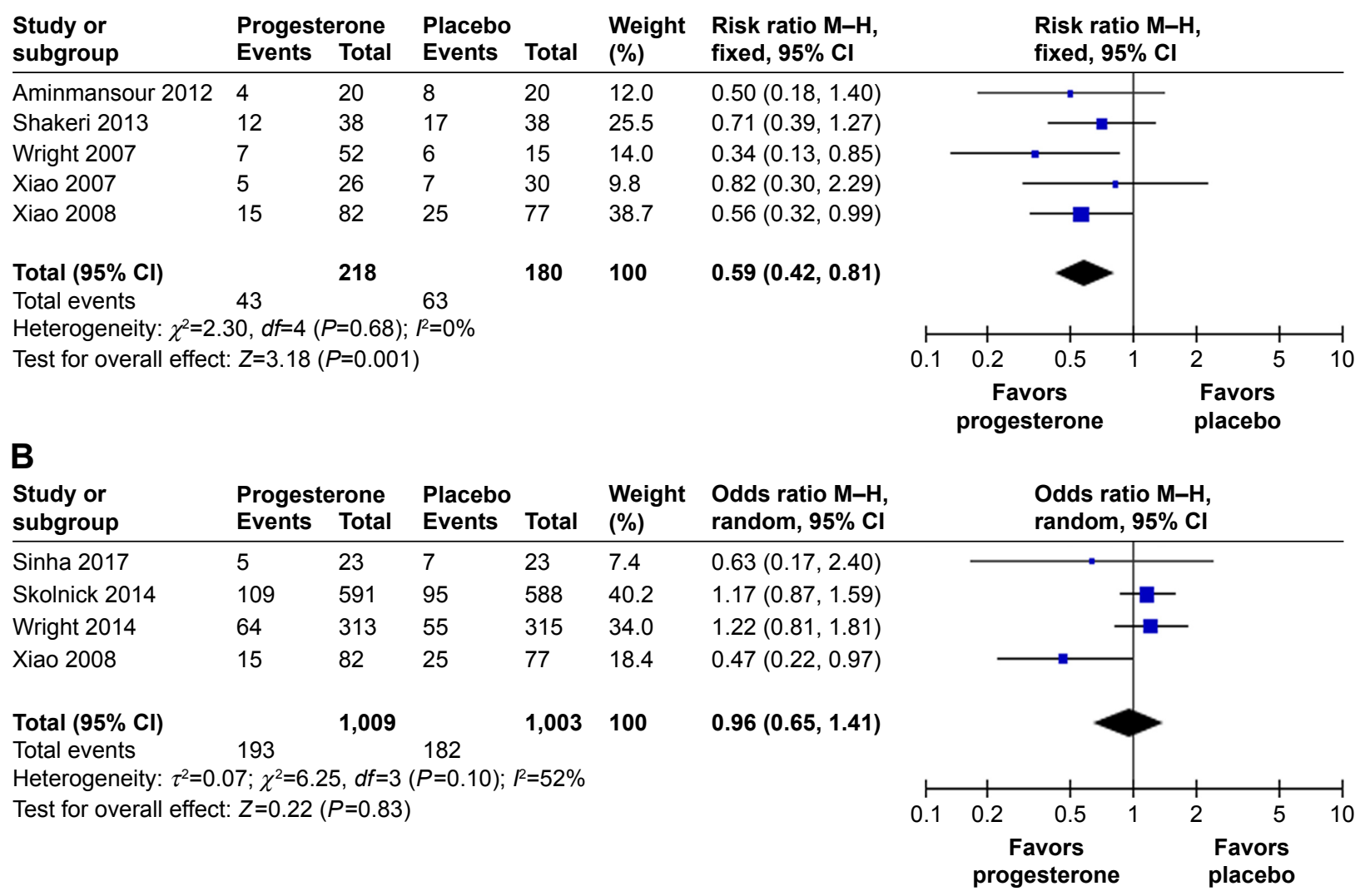

Figure 3 Forest plot showing the risk ratio of mortality within 3 months $(\mathbf{A})$ and at 6 months $(\mathbf{B})$ post-injury.

A

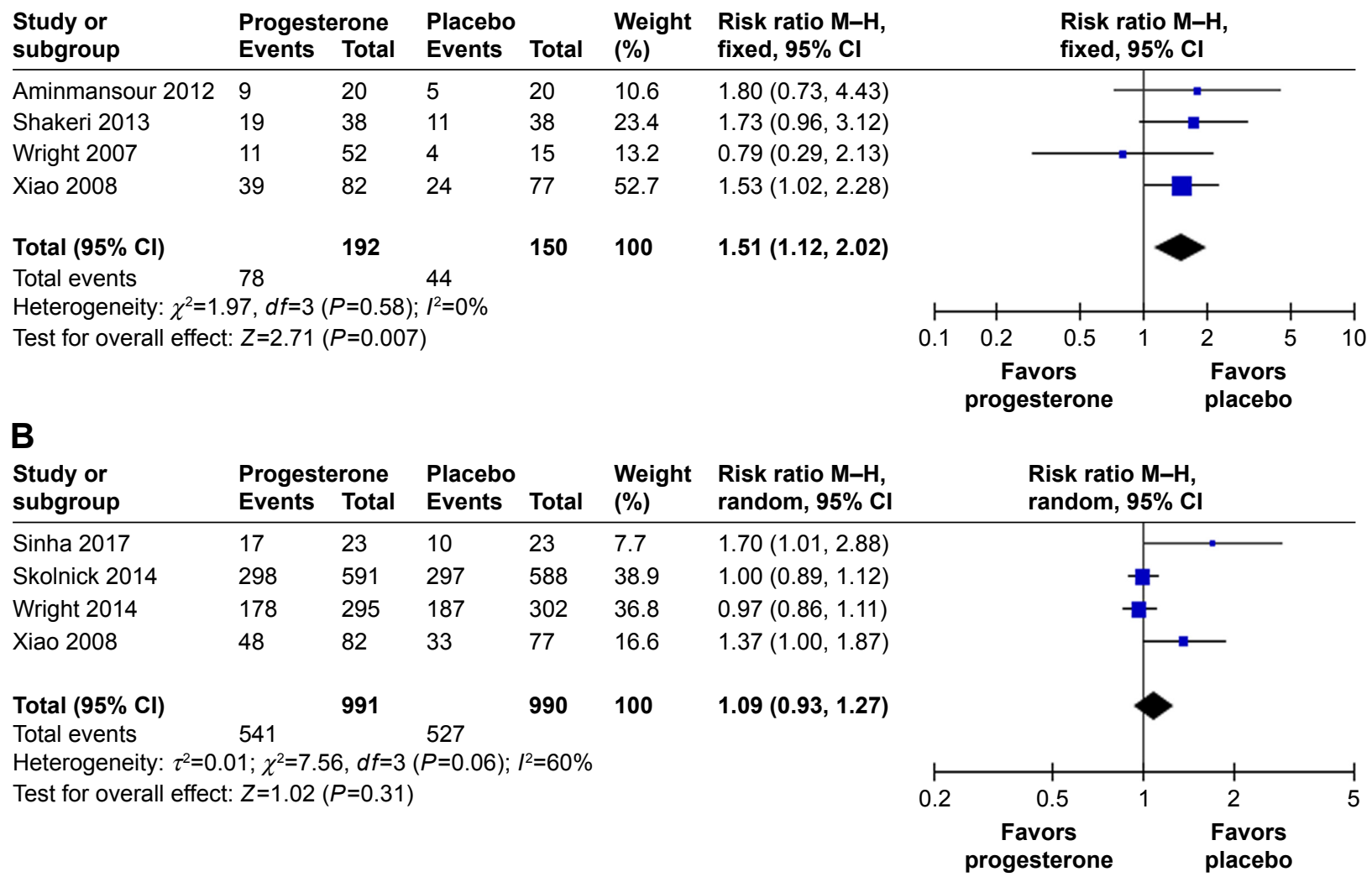

Figure 4 Forest plot showing the risk ratio of neurological outcome within 3 months $(\mathbf{A})$ and at 6 months (B) post-injury. 


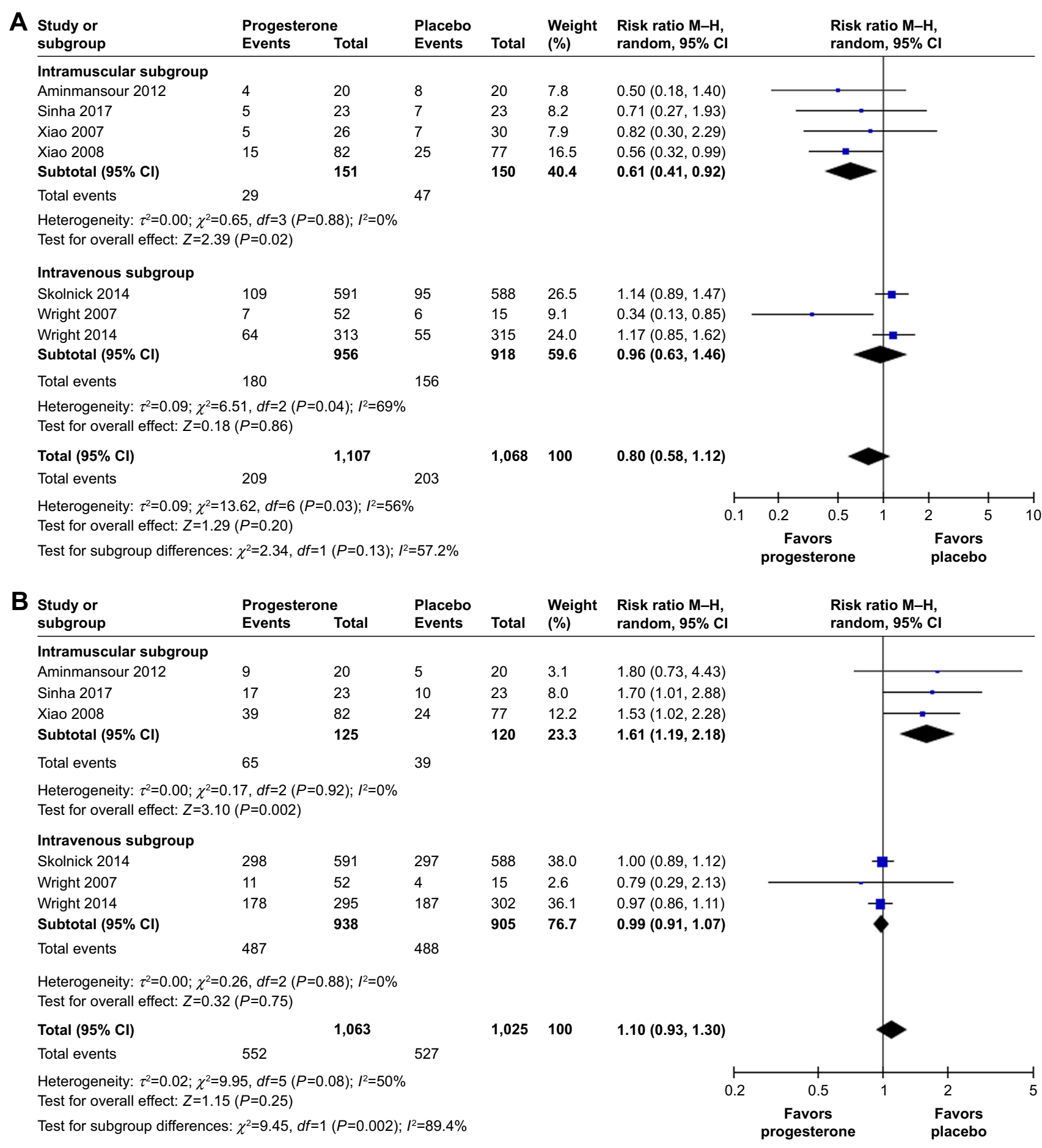

Figure 5 Forest plot showing the risk ratio of mortality $(\mathbf{A})$ and neurological outcome $(\mathbf{B})$ in patients given intramuscular or intravenous progesterone.

follow-up period and more sensitive and quantitative measures of functional recovery to confirm the long-term effects of progesterone. ${ }^{26}$

Drug dose and delivery route are important parameters that influence clinical efficacy. Of the included trials, progesterone was administered intravenously in three studies and intramuscularly in four. One study gave a medroxyprogesterone tablet by gavage via nasogastric tube. Our analysis showed that progesterone only conferred neuroprotection in patients who were given progesterone intramuscularly. The discrepancy in the efficacies of intravenous and intramuscular administration is unclear. In humans, progesterone levels in both the plasma and cerebrospinal fluid rapidly and transiently increase after severe TBI, ${ }^{27,28}$ possibly as part of the endogenous protective response..$^{29}$ The addition of exogenous progesterone may augment the physiological neuroprotective 
response. Intravenous infusion enables a rapid increase in serum concentration. Continuous intravenous administration of progesterone at a dose of $12 \mathrm{mg} / \mathrm{kg} /$ day resulted in a steady-state serum concentration of $\sim 1.1 \mu \mathrm{M}$, which is much higher than in untreated patients. ${ }^{16,17,30}$ Such a high concentration of progesterone is never reached under physiological conditions. In experimental rodent models, lower doses ( 8 and $16 \mathrm{mg} / \mathrm{kg}$ ) of progesterone were more efficient than a higher dose (32 mg/kg), suggesting an inverted U-shaped relationship between progesterone dose and neuroprotection. ${ }^{31,32}$ Thus, high circulating progesterone levels after continuous intravenous infusion may be the major reason for the differential efficacies observed in our analysis. In fact, the mismatch between preclinical and clinical studies has raised concerns about the dose, route, and duration of progesterone administration. ${ }^{26,29}$ Based on allometric scaling calculations, Howard et al proposed that doses below $8 \mathrm{mg} / \mathrm{kg} /$ day may be more effective in humans. ${ }^{33}$ Preclinical studies strongly suggest that progesterone is a promising neuroprotective agent for brain injury, but the treatment protocol needs to be modified based on further clinical trials. More investigations are necessary to clarify the pharmacological characteristics of different progesterone delivery routes. For example, intranasal delivery is a promising therapeutic option for drug delivery, especially for emergent events such as stroke and TBI. ${ }^{34}$ More effective progesterone analogs that are easier to store, transport, formulate, and administer should be explored.

In addition to dose, duration, and administration route, pre-hospital factors may also affect clinical outcomes. Denninghoff et al reported that pre-hospital intubation was associated with favorable outcomes and lower mortality in patients with moderate or severe TBI. ${ }^{35}$ However, whether pre-hospital intubation affects patient response to progesterone treatment remains unknown. Potential prehospital factors that could affect patient enrollment criteria and response to progesterone treatment should be considered in trial design and interpretation. ${ }^{36}$

\section{Limitations}

The present findings must be viewed in the context of potential limitations. Firstly, only eight studies were included for analysis. Negative or neutral studies are less likely to be published, so the results of our analysis may be overstated. Secondly, the sensitivity analysis revealed instability of the pooled estimates in the analysis of neurologic outcomes within 3 months post-injury. Removing the study by Xiao et al negated the beneficial effect of progesterone. One potential reason for this sensitivity could be the large sample in the study by Xiao et al. Thirdly, the standard dosage and duration of progesterone administration are lacking. Variation of these characteristics may influence clinical efficacy. Finally, the types of TBI in these studies were not identical. High-quality, large-scale, and rigorously designed RCTs involving different follow-up periods and administration routes are still needed to confirm the effects of progesterone in patients with severe TBI.

\section{Conclusion}

Progesterone administration improves the clinical outcomes of severe TBI patients within 3 months post-injury but may not have significant long-term benefits at 6 months post-injury.

\section{Acknowledgments}

The work was supported by grants from Hubei Province Health and Family Planning Scientific Research Project (WJ2017H0012) and Wuhan Science and Technology Bureau Research Project (2018060401011312).

\section{Disclosure}

The authors report no conflicts of interest in this work.

\section{References}

1. Menon DK, Schwab K, Wright DW, Maas AI; Demographics and Clinical Assessment Working Group of the International and Interagency Initiative toward Common Data Elements for Research on Traumatic Brain Injury and Psychological Health. Position statement: definition of traumatic brain injury. Arch Phys Med Rehabil. 2010;91(11):1637-1640.

2. Horneman G, Emanuelson I. Cognitive outcome in children and young adults who sustained severe and moderate traumatic brain injury 10 years earlier. Brain Inj. 2009;23(11):907-914.

3. Groswasser Z, Cohen M, Keren O. Female TBI patients recover better than males. Brain Inj. 1998;12(9):805-808.

4. Baulieu EE, Robel P, Schumacher M. Neurosteroids: beginning of the story. Int Rev Neurobiol. 2001;46:1-32.

5. Schumacher M, Akwa Y, Guennoun R, et al. Steroid synthesis and metabolism in the nervous system: trophic and protective effects. J Neurocytol. 2000;29(5-6):307-326.

6. Wang X, Zhang J, Yang Y, et al. Progesterone attenuates cerebral edema in neonatal rats with hypoxic-ischemic brain damage by inhibiting the expression of matrix metalloproteinase-9 and aquaporin-4. Exp Ther Med. 2013;6(1):263-267.

7. He L, Zhang X, Wei X, Li Y. Progesterone attenuates aquaporin-4 expression in an astrocyte model of ischemia/reperfusion. Neurochem Res. 2014;39(11):2251-2261.

8. Si D, Li J, Liu J, et al. Progesterone protects blood-brain barrier function and improves neurological outcome following traumatic brain injury in rats. Exp Ther Med. 2014;8(3):1010-1014.

9. Lei B, Mace B, Dawson HN, Warner DS, Laskowitz DT, James ML. Anti-inflammatory effects of progesterone in lipopolysaccharidestimulated BV-2 microglia. PLoS One. 2014;9(7):e103969.

10. Chen G, Shi JX, Qi M, Wang HX, Hang CH. Effects of progesterone on intestinal inflammatory response, mucosa structure alterations, and apoptosis following traumatic brain injury in male rats. $J$ Surg Res. 2008; 147(1):92-98.

11. Pascual JL, Murcy MA, Li S, et al. Neuroprotective effects of progesterone in traumatic brain injury: blunted in vivo neutrophil activation at the blood-brain barrier. Am J Surg. 2013;206(6):840-846. 
12. Candolfi M, Jaita G, Zaldivar V, et al. Progesterone antagonizes the permissive action of estradiol on tumor necrosis factor-alpha-induced apoptosis of anterior pituitary cells. Endocrinology. 2005;146(2):736-743.

13. Gibson CL, Constantin D, Prior MJW, Bath PMW, Murphy SP. Progesterone suppresses the inflammatory response and nitric oxide synthase-2 expression following cerebral ischemia. Exp Neurol. 2005; 193(2):522-530.

14. Wright DW, Kellermann AL, Hertzberg VS, et al. ProTECT: a randomized clinical trial of progesterone for acute traumatic brain injury. Ann Emerg Med. 2007;49(4):391-402.

15. Ma J, Huang S, Qin S, You C. Progesterone for acute traumatic brain injury. Cochrane Database Syst Rev. 2012;10:CD008409.

16. Wright DW, Yeatts SD, Silbergleit R, et al. Very early administration of progesterone for acute traumatic brain injury. N Engl J Med. 2014; 371(26):2457-2466.

17. Skolnick BE, Maas AI, Narayan RK, et al. A clinical trial of progesterone for severe traumatic brain injury. N Engl J Med. 2014;371(26): 2467-2476.

18. Bradburn MJ, Deeks JJ, Berlin JA, Russell Localio A. Much ado about nothing: a comparison of the performance of meta-analytical methods with rare events. Stat Med. 2007;26(1):53-77.

19. Xiao GM, Wei J, Wu ZH, et al. [Clinical study on the therapeutic effects and mechanism of progesterone in the treatment for acute severe head injury]. Zhonghua Wai Ke Za Zhi. 2007;45(2):106-108. Chinese.

20. Aminmansour B, Nikbakht H, Ghorbani A, et al. Comparison of the administration of progesterone versus progesterone and vitamin D in improvement of outcomes in patients with traumatic brain injury: A randomized clinical trial with placebo group. Adv Biomed Res. 2012; $1: 58$

21. Shakeri M, Boustani MR, Pak N, et al. Effect of progesterone administration on prognosis of patients with diffuse axonal injury due to severe head trauma. Clin Neurol Neurosurg. 2013;115(10):2019-2022.

22. Xiao G, Wei J, Yan W, Wang W, Lu Z. Improved outcomes from the administration of progesterone for patients with acute severe traumatic brain injury: a randomized controlled trial. Crit Care. 2008;12(2):R61.

23. Sinha S, Raheja A, Samson N, et al. A randomized placebo-controlled trial of progesterone with or without hypothermia in patients with acute severe traumatic brain injury. Neurol India. 2017;65(6):1304-1311.
24. Roof RL, Duvdevani R, Braswell L, Stein DG. Progesterone facilitates cognitive recovery and reduces secondary neuronal loss caused by cortical contusion injury in male rats. Exp Neurol. 1994;129(1):64-69.

25. Lu XY, Sun H, Li QY, Lu PS. Progesterone for traumatic brain injury: a meta-analysis review of randomized controlled trials. World Neurosurg. 2016;90:199-210.

26. Stein DG. Embracing failure: What the Phase III progesterone studies can teach about TBI clinical trials. Brain Inj. 2015;29(11):1259-1272.

27. Santarsieri M, Niyonkuru C, Mccullough EH, et al. Cerebrospinal fluid cortisol and progesterone profiles and outcomes prognostication after severe traumatic brain injury. J Neurotrauma. 2014;31(8):699-712.

28. Wagner AK, McCullough EH, Niyonkuru C, et al. Acute serum hormone levels: characterization and prognosis after severe traumatic brain injury. J Neurotrauma. 2011;28(6):871-888.

29. Schumacher M, Denier C, Oudinet JP, Adams D, Guennoun R. Progesterone neuroprotection: The background of clinical trial failure. J Steroid Biochem Mol Biol. 2016;160:53-66.

30. Wright DW, Ritchie JC, Mullins RE, Kellermann AL, Denson DD. Steady-state serum concentrations of progesterone following continuous intravenous infusion in patients with acute moderate to severe traumatic brain injury. J Clin Pharmacol. 2005;45(6):640-648.

31. Goss CW, Hoffman SW, Stein DG. Behavioral effects and anatomic correlates after brain injury: a progesterone dose-response study. Pharmacol Biochem Behav. 2003;76(2):231-242.

32. Cutler SM, Cekic M, Miller DM, Wali B, Vanlandingham JW, Stein DG. Progesterone improves acute recovery after traumatic brain injury in the aged rat. J Neurotrauma. 2007;24(9):1475-1486.

33. Howard RB, Sayeed I, Stein DG. Suboptimal dosing parameters as possible factors in the negative phase III clinical trials of progesterone for traumatic brain injury. J Neurotrauma. 2017;34(11):1915-1918.

34. Guennoun R, Fréchou M, Gaignard P, et al. Intranasal administration of progesterone: A potential efficient route of delivery for cerebroprotection after acute brain injuries. Neuropharmacology. Epub 2018 Jun 6.

35. Denninghoff KR, Nuño T, Pauls Q, et al. Prehospital intubation is associated with favorable outcomes and lower mortality in ProTECT III. Prehosp Emerg Care. 2017;21(5):539-544.

36. Sayeed I, Atif F, Espinosa-Garcia C, et al. Prehospital intubation: further confounders in trial results. Prehosp Emerg Care. 2018;22(4):535-536.
Drug Design, Development and Therapy

\section{Publish your work in this journal}

Drug Design, Development and Therapy is an international, peerreviewed open-access journal that spans the spectrum of drug design and development through to clinical applications. Clinical outcomes, patient safety, and programs for the development and effective, safe, and sustained use of medicines are the features of the journal, which

\section{Dovepress}

has also been accepted for indexing on PubMed Central. The manuscript management system is completely online and includes a very quick and fair peer-review system, which is all easy to use. Visit http://www.dovepress.com/testimonials.php to read real quotes from published authors. 